\title{
Giant Solitary Fibrous Tumor of Posterior Mediastinum: A Case Report
}

Shaoli Song ${ }^{1 *}$, Panli Li ${ }^{1}$, Qiufang Liu ${ }^{1}$, Zizhou Zhao ${ }^{2}$, Daoqiang Tang ${ }^{3}$, Xiaoguang Sun ${ }^{1}$, jianjun Liu ${ }^{1}$ and Gang Huang ${ }^{1}$

${ }^{1}$ Department of Nuclear Medicine, Ren Ji Hospital, School of Medicine, Shanghai Jiao Tong University, Shanghai, China

${ }^{2}$ Department of Radiology, Ren Ji Hospital, School of Medicine, Shanghai Jiao Tong University, Shanghai, China

${ }^{3}$ Department of Pathology, Ren Ji Hospital, School of Medicine, Shanghai Jiao Tong University, Shanghai, China

"Corresponding author: Shaoli Song, MD and PhD, Associate Chief Physician, Department of Nuclear Medicine, Ren Ji Hospital, School of Medicine, Shanghai Jiao Tong University. Shanghai-200127, China, Tel: 86-021-68383530; E-mail: shaoli-song@163.com

Received date: Dec 13, 2015, Accepted date: Jan 11, 2016, Publication date: Jan 15, 2016

Copyright: (c) 2016 Song S, et al. This is an open-access article distributed under the terms of the Creative Commons Attribution License; which permits unrestricted use; distribution; and reproduction in any medium; provided the original author and source are credited.

Abstract
A 54-year-old male was taken to a hospital after experiencing persistent back pain for a month. A spinal column
MRI showed a mass in T5-T8 fanterior edge. After four months follow-up, another MRI revealed the mass had grown
up to the site of T3-T9. An ${ }^{18} \mathrm{~F}-\mathrm{FDG}$ PET/CT imaging was underwent and the imaging showed three tumors mass
which all had a high uptake of ${ }^{18} \mathrm{~F}-\mathrm{FDG}$ locating in thorax, abdomen and pelvis, respectively. The thoracoscopic
biopsy histological revealed the mass was mesenchymal tumor and immunohistochemistry showed positive for
CD34 and CD39, that all proved the mass is solitary fibrous tumor.

Keywords: Solitary fibrous tumor; MRI; PET

\section{Introduction}

Solitary fibrous tumor is an uncommon tumor which is from dendritic mesenchymal cells [1]. It is a slow-growing neoplasm and present as an asymptomatic mass, when the mass grow up, some symptoms come up corresponding. So the diagnostic rate of the solitary fibrous tumor was usually not high [2].

\section{Case Report}

A 54-year-old male was taken to a hospital after experiencing persistent back pain for a month. He had no spine movement disorder, physical activity limitation and numbness. He also had no other chest complaints, such as cough, chest tightness, shortness of breath, or hemoptysis.

His smoking history consisted of ten cigarettes per day for the past ten years. Examination of the chest revealed coarse breath sounds and had no wet and dry rales. In January 2014, a sagittal spinal column magnetic resonance imaging (MRI) showed a giant stripped solid mass extended from the anterior edge of T5 down to T8.

The T2WI image showed a higher signal intermediate, on the contrary for the T1WI (Figure 1A). The following contrast-enhanced computed tomography (CECT) had the same result. In the plain scan, lung and mediastinal windows were used to read the images (Figure 1B and $1 \mathrm{C}$ ). For the enhanced images, from arterial to venous phase, the mass strengthened gradually (Figure 1D and 1E).

Then after four months follow-up, another MRI was performed and revealed the mass had grown up from the fanterior edge of T3 down to T9 (Figure 1F). The signal information was similar to the early one.

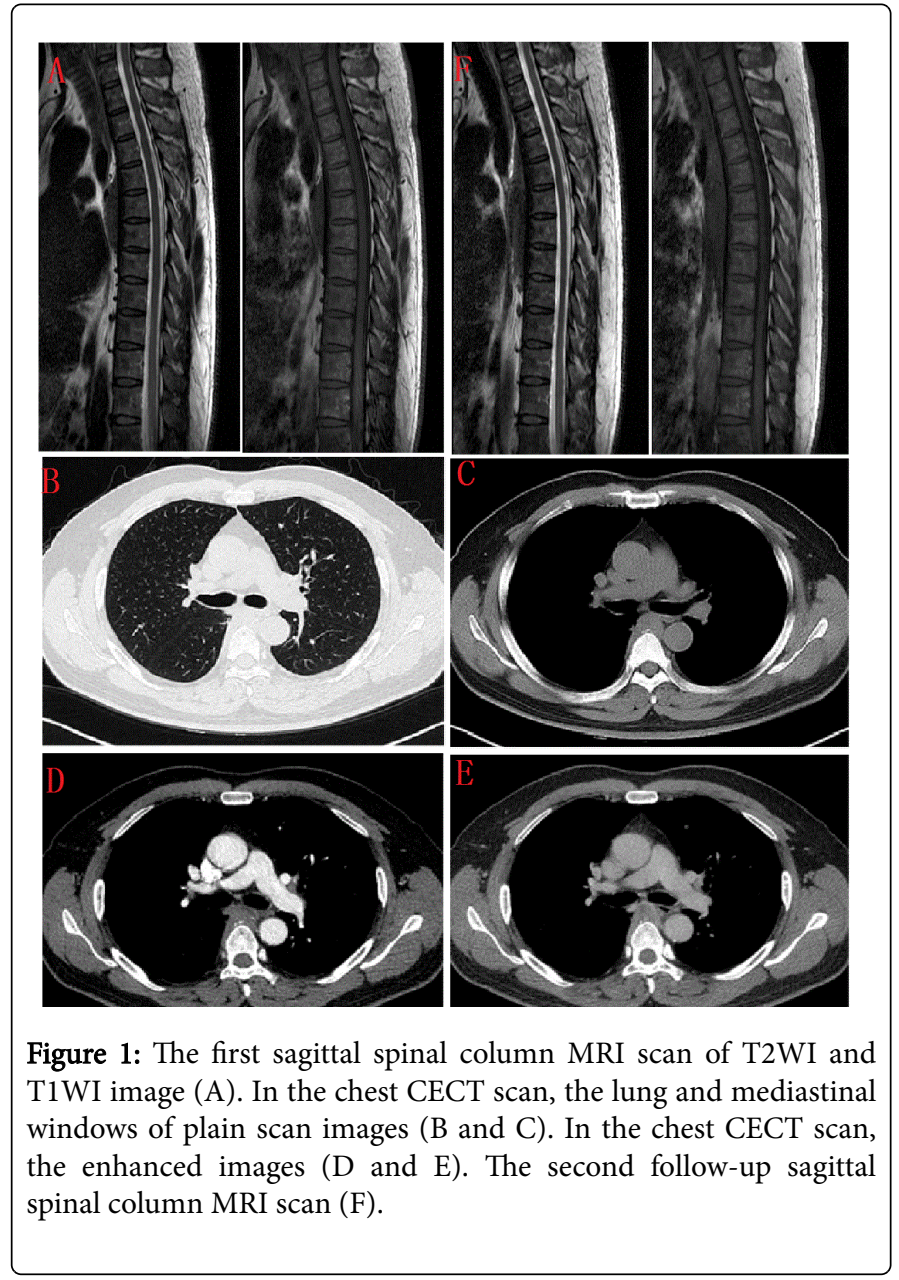

In order to definite the property of the mass, in June 2014, a fluorine-18-fluorodeoxygulucose positron emission tomography/ 
computed tomography $\left({ }^{18} \mathrm{~F}-\mathrm{FDG} \mathrm{PET} / \mathrm{CT}\right)$ was performed. In the view of transverse section, PET/CT fusion image confirmed that the mass showed a mildly increased FDG uptake with standardized uptake value (SUV) max of 8.8 (Figure 2A). Near to the right arteria iliaca communis saw thickening soft tissue density with rough border, measuring $4 \times 3.2 \times 4.9 \mathrm{~cm}$, SUV max of 6.8 (Figure 2B). Beside the colon sigmoideum also had a hypermatabolic activity area with SUV $\max$ of 7.1 and size of $5 \times 4.6 \times 4.6 \mathrm{~cm}$ (Figure 3C).

On 7 July 2014, a thoracoscopic biopsy histological was taken for the lesion in the fanterior edge of thoracic. Photomicrographs (Figure 3A, hematoxylin-eosin, original magnification 40×; Figure $3 \mathrm{~B}$, hematoxylin-eosin, original magnification $100 \times$ ) showed uniform spindled cells revealing the mass was mesenchymal tumor. The immunohistochemistry results showed that the tumor cells were negative for SMA, VIM and S-100, positive for CD34 and CD99 (Figure $3 \mathrm{C}$ and $3 \mathrm{D}$ ). The mass was proved to be solitary fibrous tumor.
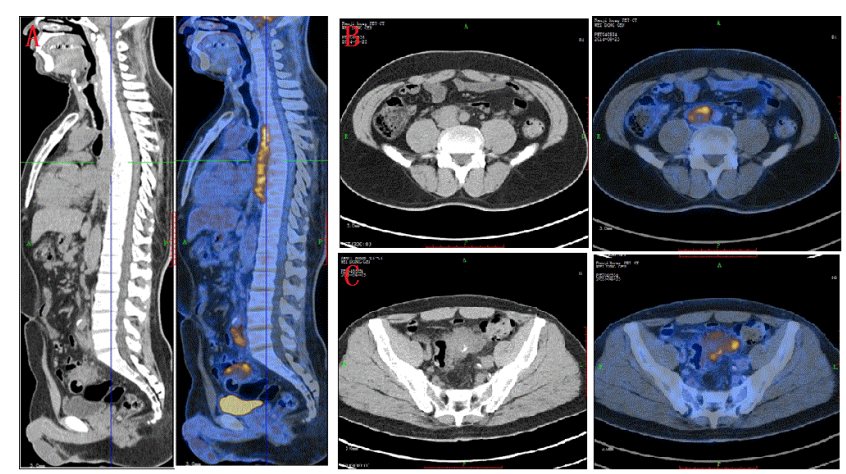

Figure 2: PET/CT fusion image showed the SUV information about the mass (A). Near to the right arteria iliaca communis there was a thickening soft tissue (B). The image showed that beside the colon sigmoideum there was a soft tissue (C).

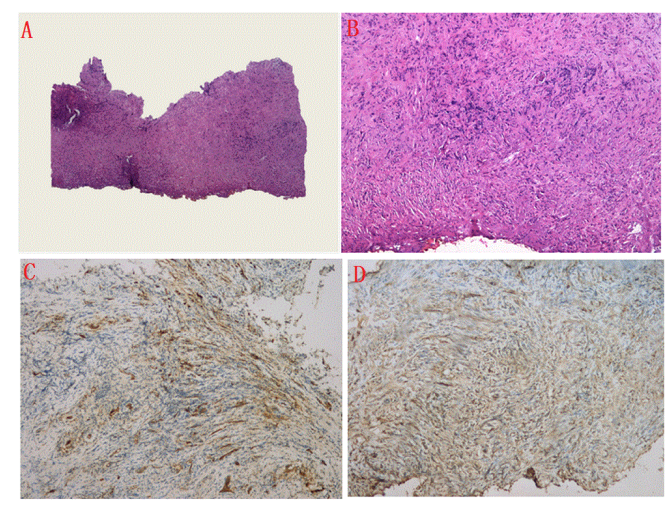

Figure 3: The photomicrographs of the biopsies for the mass (A, hematoxylin-eosin, original magnification $40 \times$; B, hematoxylineosin, original magnification $100 \times$ ). The immunohistochemistry results that the mass had positive for CD34 and CD99 (C and D).

\section{Discussion}

A solitary fibrous tumor is a rare form of tumor that is only occasionally cited in the literature, usually in single cases. In most solitary fibrous tumor cases, CECT may show heterogeneous density, so as the MRI signal information. Traditional imaging methods are not sensitive for the diagnosis of solitary fibrous tumors [3,4].Though solitary fibrous tumors are benign tumors, about $20 \%$ of solitary fibrous tumors have maligant potential [5]. To the case in the paper, we find three focus in the patient. MRI scan as a partial imaging of the body only find one lesion, but PET/CT as a whole body scan find other two lesions. PET/CT can provide the matabolic and the whole body information that has its unique advantages in functional diagnosis [6]. In addition, some studies show that when the tumor has the maligant potential, the mass will have a high FDG uptake [7]. So the FDG uptake degree may be one factor of the tumor's aggressive behaviour [8].

In our case, the tumors have a moderate high FDG uptake in accordance with the maligant degree. Through appropriate biopsy get the histological and the immunohistochemistry result is the gold standard of the solitary fibrous tumor disgnostic [1-9]. Surgery is the mainstay of treatment, and recurrence and the clinical outcome is mainly related to the completeness of the surgical treatment $[10,11]$.

\section{Acknowledgments}

This work was funded by the National Natural Science Foundation of China (grants No. 81471708), and Shanghai Jiao Tong University Medical Engineering Cross research fund (No.YG2012MS13). No other potential conflict of interest relevant to this article was reported.

\section{References}

1. Hanau CA, Miettnen M (1995) Solitary Firous tumor: histological and immunohistochemical spectrum of benign and malignant variants presenting at diffrent sites. Hum Pathol 26: 440-449.

2. England DM, Hochholzer L, McCarthy MJ (1989) Localized benign and malignant firous tumors of the pleura: a clinicopathologic review of 223 cases. Am J Surg Pathol 13: 640-658.

3. Cardillo O, Facciolo F, Cavazzana AO, Capece G, Gasparri R, et al. (2000) Localized (solitary) fibrous tumours of the pleura: an analysis of 55 patients. Ann Thorac Surg 70: 1808-1812.

4. Versluis PJ, Lamers RJS (1994) Localized pleural fibroma: radiological features. Eur J Rad 18: 124-125.

5. Gold JS, Antonescu CR, Hajdu C, Ferrone CR, Hussain M, et al. (2002) Clinicopathologic correlates of solitary fibrous tumors.Cancer 94: 1057-1068.

6. Beadsmoore C, Newman D, Maclver D, Pawaroo D (2015) Positron Emission Tomography Computed Tomography: A Guide for the General Radiologist. Can Assoc Radiol J 66:332-347.

7. Wakisaka N, Kondo S (2009)A solitary fibrous tumor arising in the parapharyngeal space, with MRI and FDG-PET findings. Auris Nasus Larynx 36: 367-371.

8. Aisheng D, Changjing Z, Yang W, Yong C (2014) Enhanced CT and FDG $\mathrm{PET} / \mathrm{CT}$ in Malignant Solitary Fibrous Tumor of the Lung. Clin Nucl Med 39: 488-491.

9. Weynand B, Noel H, Goncette L, Noirhomme P, Collard P (1997) Solitary fibrous tumor of the pleura: a report of five cases diagnosed by transthoracic cutting needle biopsy. Chest 112: 1424-1428.

10. Perrot MD, Kurt AM, Robert JH, Borisch B, Spiliopoulos A (1999) Clinical behaviour of solitary tumours of the pleura-eight new cases and review of 360 cases in the literature. Ann Thorac Surg 67: 1456-1459. 
Citation: Song S, Li P, Liu Q, Zhao Z, Tang D, et al. (2016) Giant Solitary Fibrous Tumor of Posterior Mediastinum: A Case Report. J Nucl Med Radiat Ther 7: 276. doi:10.4172/2155-9619.1000276

Page 3 of 3

11. Sung SH, Chang JW, Kim J, Lee KS, Han J, et al. (2005) Solitary fibrous tumors of the pleura: Surgical outcome and clinical course. Ann Thorac Surg 79: 303-307. 